\title{
High-Frequency $\gamma$-Band Activity in the Basal Temporal Cortex during Picture-Naming and Lexical-Decision Tasks
}

\author{
Kazuyo Tanji, ${ }^{1,2}$ Kyoko Suzuki, ${ }^{2}$ Arnaud Delorme, ${ }^{3}$ Hiroshi Shamoto, ${ }^{4}$ and Nobukazu Nakasato ${ }^{4}$ \\ ${ }^{1}$ Graduate School of International Cultural Studies and ${ }^{2}$ Department of Behavioral Neurology and Cognitive Neuroscience, Tohoku University, Sendai 980- \\ 8576, Japan, ${ }^{3}$ Computational Neurobiology Laboratory, Salk Institute for Biological Studies, La Jolla, California 92037, and ${ }^{4}$ Department of Neurosurgery, \\ Kohnan Hospital, Sendai 982-8523, Japan
}

\begin{abstract}
$\gamma$-Band activity (GBA) in electroencephalograms (EEGs) has been shown to reflect various cognitive processes. GBA has typically been recorded in the 30-60 Hz range in scalp EEGs. Recently, task-related "high GBA" (HGBA) with frequencies up to $100 \mathrm{~Hz}$ has been observed in studies with invasive electrocorticograms (ECoGs). In the present study, we recorded ECoGs from the bilateral basal temporal cortices in a patient with epilepsy and evaluated the task-related HGBA (most prominently in the $80-120 \mathrm{~Hz}$ range) accompanying picture-naming and lexical-decision tasks. We examined picture naming using two categories (line drawings of animals and tools). The lexical-decision task was performed using words and pseudowords of two distinct Japanese writing forms, kanji (morphograms) and kana (syllabograms). Task-related HGBA was observed bilaterally during the naming task. Recordings from some electrodes revealed significant differences in HGBA between animal and tool pictures. In contrast to the naming task, there was apparent left dominance in the lexical-decision task. Furthermore, significant differences in HGBA were observed between the Japanese kanji and kana words and between the kanji words and kanji pseudowords. A number of differences in the HGBA observed in the recordings from the basal temporal area were consistent with previous findings from neuroimaging and patient studies and suggest that HGBA is a good correlate of visual cognitive functions.
\end{abstract}

Key words: dyslexia; $\gamma$; language; visual word form area; fusiform gyrus; ERSP; kanji; fMRI; oscillatory activity; LFP; ECoG; naming

\section{Introduction}

There is growing evidence that localized $\gamma$-band activity (GBA) correlates with various cognitive processes. Increased GBA has been reported to accompany perception (Fries et al., 1997) and is proposed to serve as a carrier signal for "binding" of the distributed representation of sensory information, making possible the representation of an integrated whole (Gray et al., 1989). It has also been shown to be related to cognitive functions, such as attention (Fries et al., 2001) and recognition memory (Fell et al., 2001).

GBA recorded in animals ranges in frequency from 30 to $>100 \mathrm{~Hz}$ (Gray et al., 1992; Eckhorn et al., 1993; Fries et al., 2001). However, many scalp electroencephalogram (EEG) studies in humans have reported a relatively narrow GBA in the $30-60 \mathrm{~Hz}$ range, perhaps because higher-frequency components are strongly attenuated in extracranial recordings because of spa-

\footnotetext{
Received Aug. 5, 2004; revised Jan. 26, 2005; accepted Feb. 6, 2005.

This work was supported by the 21st Century Center of Excellence Program (Ministry of Education, Culture, Sports, Science, and Technology) entitled "A Strategic Research and Education (enter for an Integrated Approach to Language and Cognition" (Tohoku University). We thank J. Riera, E. Düzel, and M. Mishkin for discussion and critical reading of this manuscript, E. Mori and J. Tanji for discussion and support, and T. Miyamoto for comments on Japanese writing systems. We thank A. Kanno, S. Sakamoto, F. Saito, Y. Suzuki, M. Iwasaki, J. Nenonen, T. Miyashita, K. Sekiya, K. Yamashita, P. Simos, A. Papanicolaou, and participants on the EEGLAB mailing list for technica assistance.

Correspondence should be addressed to Kazuyo Tanji, National Institute of Mental Health, Laboratory of Neuropsychology, Building 49, Room 1B-80, Bethesda, MD 20892. E-mail: tanjik@mail.nih.gov.

D0I:10.1523/JNEUROSCI.4948-04.2005

Copyright $\odot 2005$ Society for Neuroscience $\quad$ 0270-6474/05/253287-07\$15.00/0
}

tial summation and phase cancellation (Pfurtscheller and Cooper, 1975). Recently, Crone et al. (2001) reported auditory eventrelated power augmentation in a broader $\gamma$-band in human intracranial electrocorticograms (ECoGs), ranging $>100 \mathrm{~Hz}$, most prominently between 80 and $100 \mathrm{~Hz}$. The authors showed that this activity, named "high- $\gamma$ " activity, occurred in a more discrete and specific spatial pattern than did changes in lower- $\gamma$ band and $\alpha$ desynchronization. They proposed that high- $\gamma$ power augmentation might serve as an index of task-related cortical activation (Crone et al., 1998).

The objective of this study was to determine whether and how the high $\gamma$-band activity (HGBA) reflected functional specialization during reading and naming in a Japanese subject. We recorded electrocorticographic oscillatory activity in the bilateral basal temporal cortices of an epilepsy patient while the patient performed a lexical-decision task (reading visually presented words) and a naming task. The Japanese writing system differs from alphabetic systems in that it has two qualitatively different scripts: logographic and morphographic kanji, derived from Chinese characters, and syllabic kana. The lexical-decision task design thus manipulated two factors: (1) whether the stimulus was presented in kanji or in kana and (2) whether the stimulus was a word or a pseudoword. Our predictions were threefold. (1) Given previous reports suggesting the importance of the basal temporal area for kanji processing (Tokunaga et al., 1999), there would be greater HGBA recorded from the left basal temporal area in response to kanji than that recorded in response to kana. (2) A 
difference in responses to words and pseudowords would be seen in the HGBA, as has been seen in neuroimaging studies of this brain area (Mechelli et al., 2003). (3) Some commonality between object naming and kanji reading would be apparent in the HGBA recordings, as has been suggested by studies of brain-damaged patients and by an electrocorticographic stimulation study (Sakurai et al., 1994; Usui et al., 2003).

\section{Materials and Methods}

Subject

A 24-year-old right-handed man with intractable epilepsy was evaluated for surgical treatment. At 18 years of age, he began to have complex partial seizures, which occasionally developed into secondary generalized seizures. The seizures were refractory to medical treatment, and he was referred to our hospital for surgical consultation. The neurological examination was unremarkable. The Wechsler Adult Intelligence ScaleRevised revealed a verbal intelligence quotient (IQ) of 87, performance IQ of 82, and full-scale IQ of 82. Magnetic resonance imaging showed a small cortical dysplasia in the right frontal subcortex. Scalp EEG detected epileptiform activities from the left frontal and right temporal areas. Ictal single-photon emission tomography indicated hypoperfusion in the left temporal area. Consequently, subdural grid implantation was indicated for localization of the epileptic foci and for functional mapping of the motor, sensory, and language areas. Signed informed consent was obtained after a detailed explanation of the ECoG and language evaluation. Subdural grid electrodes were located bilaterally in the lateral and basal temporal areas. The exposed surface of each electrode was $4 \mathrm{~mm}$ in diameter, and the interelectrode spacing was $10 \mathrm{~mm}$. By stimulation assessment, the patient's language function was determined to be leftlateralized. ECoG analysis revealed that the patient's epileptic focus was centered in the left parahippocampal gyrus, recorded from two electrodes: one medial to channel 6 and the other medial to channel 7 (the activities from these electrodes were excluded from the analysis) (see Fig. 4).

\section{Stimuli and tasks}

We recorded the ECoG while the patient performed a lexical-decision task in kanji and kana and a silent object-naming task. The same tasks were performed on 2 consecutive days. The recordings were made mainly from the lateral temporal areas on day 1 and mainly from the basal temporal area on day 2 . The patient was seated in a dimly lit, magneticshielded room designed for magnetoencephalography recording. A computer monitor (refresh rate, $75 \mathrm{~Hz}$ ) was placed $90 \mathrm{~cm}$ from the patient. All visual stimuli were delivered in random order for $0.5 \mathrm{~s}$ at the center of the monitor, with an interstimulus interval of $3.5 \mathrm{~s}$.

Lexical-decision tasks. The stimuli for the word-reading task consisted of two-character kanji words and kanji pseudowords and two- or threecharacter kana words and pseudowords; 40 stimuli of each type were presented. Meaningless kanji pseudowords were formed with component characters used in real kanji words (Fig. 1, top strand). Kana pseudowords were formed by transposing syllable characters used in meaningful kana words (Fig. 1, bottom strand). A total of 160 stimuli were mixed randomly, and each stimulus was presented twice, yielding 320 ( 80 for each condition) data epochs. All character strings were viewed as white characters against a black background. The patient was requested to silently read them and judge whether they were real words. Behavioral data were collected in the same setting off-line after the recording. The patient scored $97.5 \%$ correct responses for the kanji tasks and $97.5 \%$ for the kana tasks.

Naming tasks. The stimuli for the picture-naming task consisted of 80 black-and-white line drawings. One-half of the line drawings were of animals (mammals, birds, fish, and insects), and the other half were of man-made tools. The word frequency was matched between the subcategories. The pictures were randomly mixed, and each was presented twice. The patient was requested to silently name each represented object. Behavioral data were collected in the same way as in the lexicaldecision task. The patient scored $100 \%$ for this task.

Auditory-recognition tasks. As part of a separate experiment, an auditory word-recognition task was performed according to the method of Breier et al. (1999). The patient learned 30 target words before the ECoG

\begin{tabular}{|c|c|c|c|}
\hline Word & $\begin{array}{l}\text { 毎日” } \\
\text { 主人 }\end{array}$ & $\begin{array}{l}\text { (daily) } \\
\text { (host) }\end{array}$ & $\begin{array}{l}\text { /mai-nichi/ } \\
\text { /shu-jin/ }\end{array}$ \\
\hline $\begin{array}{l}\text { Pseudo- } \\
\text { word }\end{array}$ & 日主 & $(-)$ & $\begin{array}{l}\text { /hi-nushi/, /nichi-shu/ } \\
\text { /hi-shu/, /nichi-nushi/, etc. }\end{array}$ \\
\hline
\end{tabular}

\section{kana}

Word

なまえ (name) /na-ma-e/

Pseudoword

まえな゙ (-) /ma-e-na/

Figure 1. Examples of kanji words, pseudowords, kana words, and kana pseudowords. Kanji pseudowords were formed from component characters used in real kanji words (arrowheads). Note that kanji pseudowords have multiple possible readings. In kana pseudowords, the syllable $/ \mathrm{na} /$ (arrow) in the real word "na-ma-e" was transposed to form the pseudoword "ma-e-na."

recording. A list of 70 recorded word stimuli (duration, $600 \mathrm{~ms}$ ) was presented at an $80 \mathrm{~dB}$ sound pressure level bilaterally using ear tubes, in four blocks of 40 words. Each block consisted of the 30 target words randomly mixed with 10 distractors that were unique to each list, for a total of 160 events. During the recording, the patient was asked to silently judge whether the presented words were new or old (i.e., target words). This task was performed only on day 1, with limited electrodes placed in the basal temporal area.

\section{Data analysis}

The ECoG was recorded with a 64-channel EEG system (Neuromag, Helsinki, Finland) using an intracranial reference, which was later rereferenced to an average electrode, placed in the anterior middle temporal area. The signals were digitized at $1000 \mathrm{~Hz}$ and recorded to a computer hard disk along with markers for the triggers. The bandpass filter for data acquisition was set to $0.10-330 \mathrm{~Hz}$. Any trials containing epileptic discharges or other artifacts were eliminated from additional analyses. The event-related potentials (ERPs) were calculated by averaging the epochs after low-pass filtering at $40 \mathrm{~Hz}$. The baseline offset ( -1 to $0 \mathrm{~s})$ was corrected after averaging.

Event-related spectral perturbation (ERSP) measures the average dynamic changes in amplitude of the broadband EEG frequency spectrum as a function of time relative to an experimental event (Makeig, 1993). The ERSP is calculated by computing the power spectrum over a sliding latency window and then averaging across the data epochs. The color of each pixel in the generated spectral image then indicates the power relative to baseline (in decibels) at a given frequency and latency. To visualize the power changes across the frequency range, the mean baseline logpower spectrum is subtracted from each spectral estimate, producing the baseline-normalized ERSP (Delorme and Makeig, 2004). Time-frequency analyses were conducted using three-cycle standard Morlet wavelets at each frequency from 6 to $140 \mathrm{~Hz}$ and progressing through the $3 \mathrm{~s}$ data epochs (from $1 \mathrm{~s}$ before to $2 \mathrm{~s}$ after stimulus onset) in $12 \mathrm{~ms}$ steps. The ERSP plots showed time-frequency points at which the mean log power was significantly higher or lower (bootstrap, $p<0.00025$ ) than the mean power during the $1 \mathrm{~s}$ prestimulus baseline period of the same epochs. For an overall view, the band-limited power changes in the $\alpha-\beta$ band $(8-14 \mathrm{~Hz})$ and high- $\gamma$ band $(80-120 \mathrm{~Hz})$ were calculated using the same wavelet decomposition method [we called it event-related synchronization (ERS) or desynchronization (ERD), following the nomenclature by Pfurtscheller and Aranibar (1979)].

To determine significant differences between two series of time-frequency images (one per trial), all single-trial spectral decomposition two-dimensional (2-D) images of the two series were pooled and then repeatedly and randomly shuffled and partitioned into two series of the same size as that of the original series. Applying the original procedure to these shuffled series [i.e., for the time-frequency decomposition, a sim- 

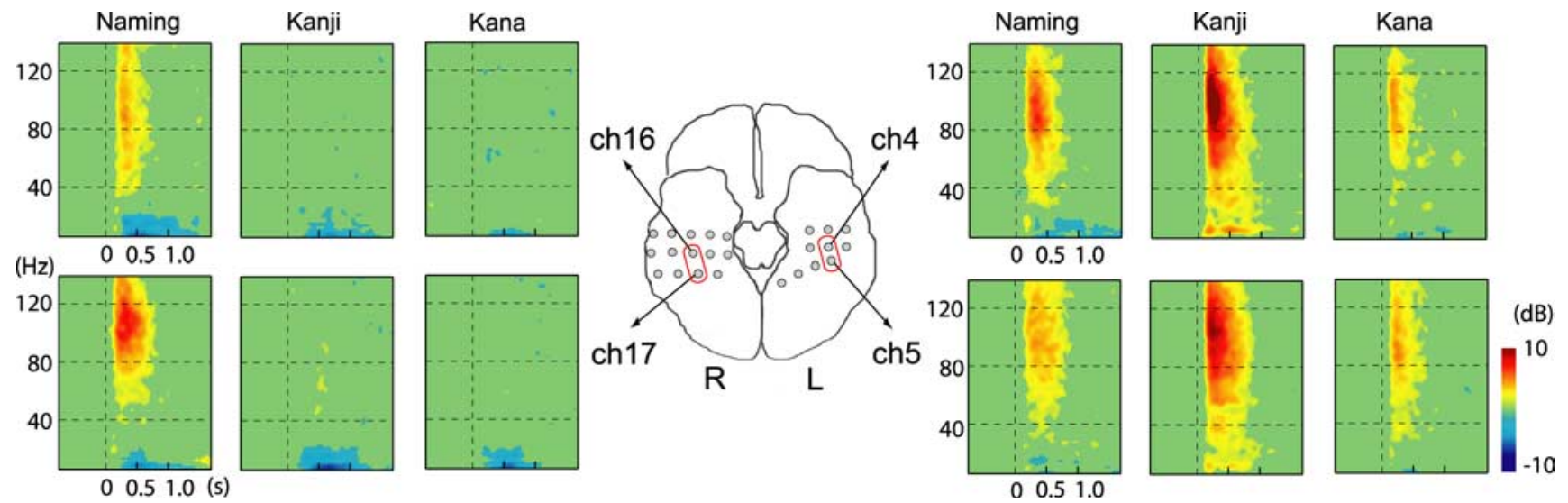

Figure 2. ERSP time-frequency plot for three conditions at representative channels [ 4 and 5 in the left (L); 16 and 17 in the right (R)] in the bilateral fusiform cortices. Nongreen areas in the time-frequency plane indicate significant poststimulus increases or decreases in the log-spectral power relative to the mean power in the averaged $1 \mathrm{~s}$ prestimulus activities, masked by bootstrap $(p<0.00025)$. In contrast to the bilateral activation by object naming, clear left laterality in activation was shown for the lexical-decision task (both kanji and kana).

ple mean of the time-frequency decomposition of single trials (Delorme and Makeig, 2004)] generated a probability distribution of ERSP values at each time-frequency point. For each time-frequency point, if the measurement from the nonshuffled data lay within this distribution, then the two series were not considered to be significantly different. This method is a 2-D extension of the original 1-D method described by Blair and Karniski (1993). As for the multiple comparisons, dividing by the total number of the time-frequency estimate in the 2-D time-frequency image decomposition would have been too conservative, because spectral estimates of neighboring time-frequency points are highly correlated. Therefore, we attempted to estimate the number of independent timefrequency points in the image. First, we used geometrical wavelets, which are optimal in terms of image compression, so that neighboring frequencies can be assumed to carry independent spectral estimates. Consequently, we had time-frequency decompositions at only seven frequencies $(120,60,30,15,7.5,3.25$, and $1.625 \mathrm{~Hz})$. For each frequency, we then found the minimum number of time points for which there was a significant correlation of the spectral estimates between neighboring time points [for each frequency and number of time point, we computed the correlation from 0 to 1 for all of the data channels (chs) to obtain a probability distribution of correlation; we then fitted this distribution using a fourth-order curve (Ramberg et al., 1979) and assessed the probability of significance for the value 0 (no correlation) to be within the distribution of the estimated correlation]. For instance, using 28 time points at $120 \mathrm{~Hz}$, there was no significant $[p>0.05$ before incorporating Bonferroni's (1950) correction for multiple comparisons] correlation between neighboring time-frequency power estimates, but there was a significant correlation using 32 time points instead of 28. Applying the same approach for the seven geometrical frequencies and summing the minimum number of time points for observing a significant correlation at all frequencies, we obtained $\sim 200$ independent estimates (to be more precise, an average of $188 \pm 13$, in which the mean and SD were obtained by applying this method to the different datasets used in this experiment). Therefore, in all of the time-frequency plots, we used a significance mask at $p<0.00025$ (0.05 of 200). An alternative method for correcting for multiple comparisons was presented by Nichols and Holmes (2002).

\section{Results}

\section{Lexical-decision task}

Time-frequency power analysis

The time-frequency plots, or ERSPs, revealed broadband highfrequency activities centered at $60-140 \mathrm{~Hz}$ (chs 4 and 5) (Fig. 2). Event-related decreases in power (desynchronizations) were seen on recordings from many electrodes in frequency bands $<20 \mathrm{~Hz}$ but not in higher-frequency bands (Fig. 2, supplemental Fig. 1, available at www.jneurosci.org as supplemental material). A sig-
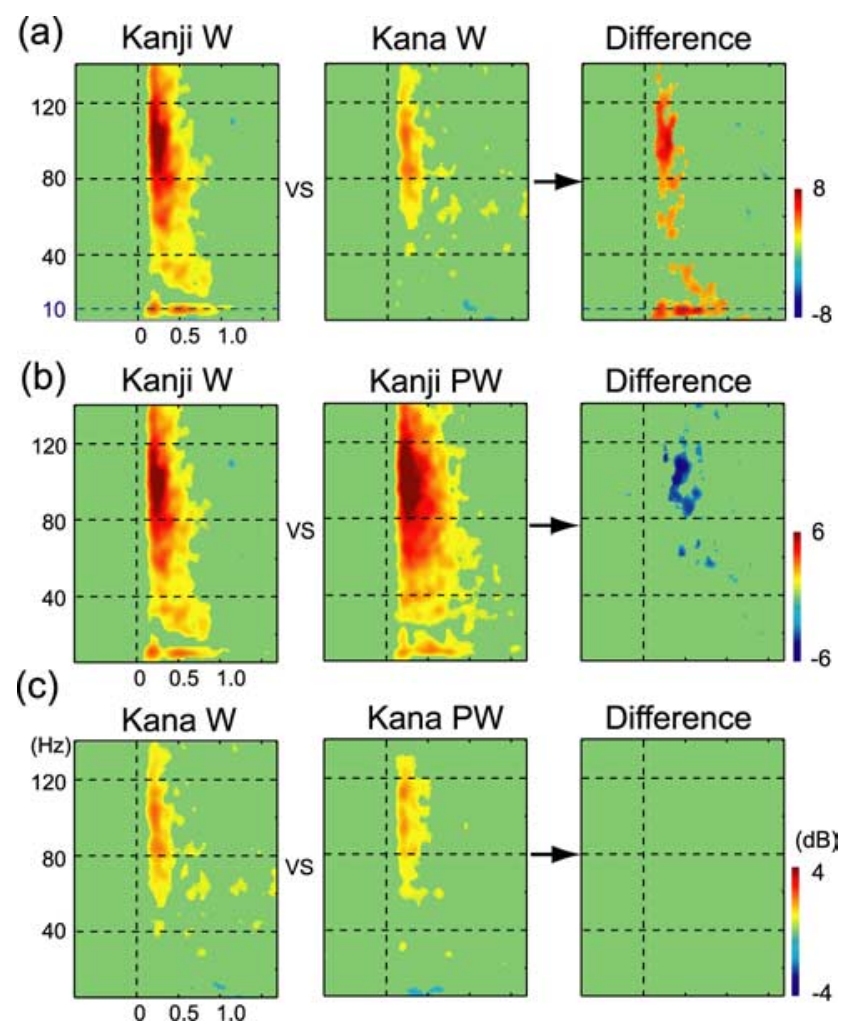

Figure 3. Comparison of ERSP plots for conditions in the lexical-decision task in channel 4. Time-frequency points with significant differences (bootstrap, $p<0.00025$ ) are shown in the right row. $\boldsymbol{a}-\boldsymbol{c}$, Comparisons were made between a kanji word (W) versus a kana W ( $\boldsymbol{a})$, a kanji $W$ versus a kanji pseudoword (PW; $\boldsymbol{b})$, and a kana W versus a kana PW (c). Differences in GBA between the kanji W and the kana W were seen in broadband spanning, including a band centered at $10 \mathrm{~Hz}$ for kanji conditions. Differences in GBA between the kanji W and PW were restricted to a higher band of $>60 \mathrm{~Hz}$. No significant difference between the kana $\mathrm{W}$ and the kana PW was observed. The color scales are common to all conditions, except for Difference plots.

nificant difference in ERSPs (bootstrap, $p<0.00025$ ) was observed between real kanji words and real kana words in broadfrequency bands, including a prominent $8-14 \mathrm{~Hz}$ band (Fig. $3 a$ ). The difference between kanji words and kanji pseudowords was restricted to higher-frequency bands $(60-120 \mathrm{~Hz})$ (Fig. 3b). No significant difference in ERSP was observed between kana words and kana pseudowords (Fig. 3c). The overall view of the ERS 

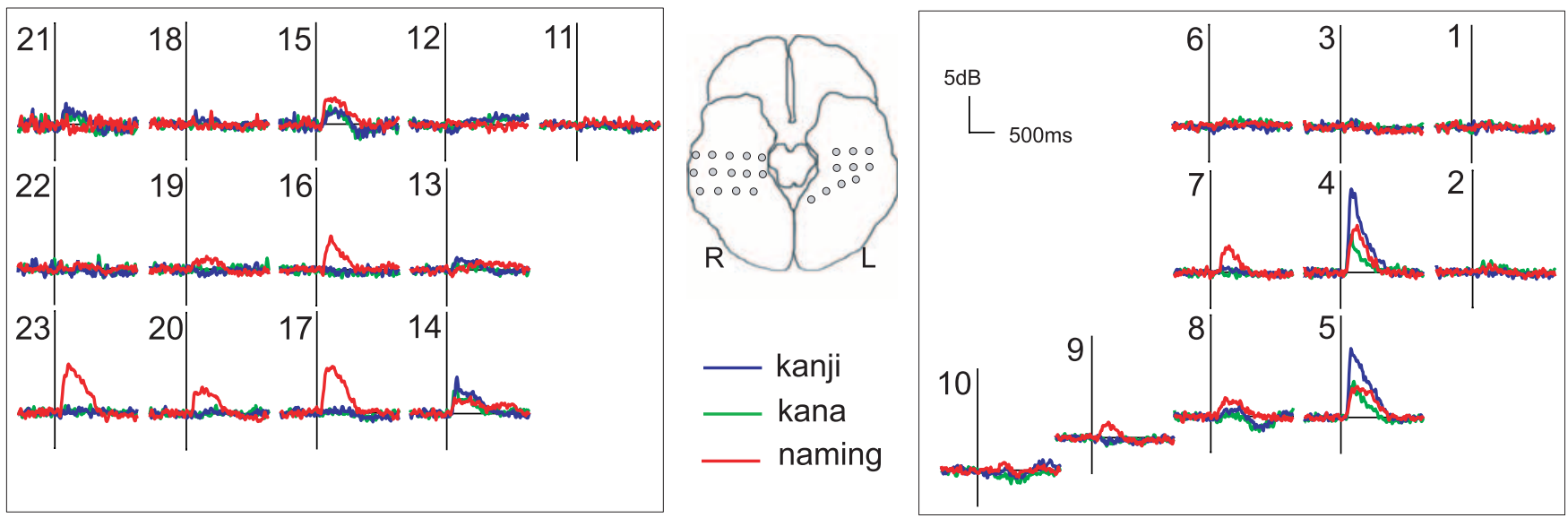

Figure 4. Power changes (ERS) in the $80-120 \mathrm{~Hz}$ band recorded from electrodes located in the bilateral basal temporal areas for three conditions: kanji, kana, and naming. Clear left laterality in the activation was shown for the lexical-decision task (both kanji and kana). Dissociation in power was observed between kanji and kana in channels 4 and 5 . Bilateral activation was seen in the naming task. R, Right; L, Left.

revealed the induced responses on selected electrodes, with clear contrasts between adjacent electrodes (Fig. 4).

\section{ERP}

Triphasic ERPs with deflections corresponding to P150, N200, and P290, as reported by Allison et al. (1999), were clearly seen with kanji stimuli (Fig. 5). Left laterality was not observed in the N200 ERPs. The N200 deflections for kanji stimuli were highest in ch 4 on the left side and in ch 14 on the right. N200 ERPs for kana were small in ch 5 and not visible in chs 4 and 14. The N200 latency for the kanji stimuli corresponded approximately to the peak timing of ERS (Fig. 5). In contrast to the large baseline fluctuations in ERP, the power changes were nearly flat before and after the task-related change (Figs. 4, 5).

\section{Picture-naming tasks}

\section{Time-frequency power analysis}

In the ERSP plots, high oscillatory activity peaking at $\sim 60-120$ $\mathrm{Hz}$ and deactivation in the lower-frequency band, similar to that seen in the lexical-decision tasks, were seen bilaterally (Fig. 2). Category-dependent double dissociation (animals vs tools) of HGBA was observed in chs 4 and 8 (Fig. 6).

The overall view of the ERS showed a bilaterally distributed positive power change in the $80-120 \mathrm{~Hz}$ band in the picturenaming tasks (Fig. 4). In the left hemisphere, the largest power change was recorded with the same two electrodes as for lexicaldecision tasks (chs 4 and 5); in ch 4, the ERS observed with the naming tasks was larger than that observed with kana stimuli. ERD was seen on recordings from many electrodes in frequency bands $<20 \mathrm{~Hz}$ but not in higher-frequency bands (Fig. 2, supplemental Fig. 1, available at www.jneurosci.org supplemental material). Results were reproducible between days 1 and 2 for the recordings from electrodes 1, 2, 3, 4, 18, 19, 21, and 22 (Fig. 7a).

\section{ERP}

N200 ERPs were seen most notably in ch 8 on the left and ch 16 on the right. There was no apparent categorical difference in the ERPs between animals and tools. The amplitudes of N200 ERPs elicited by the picture-naming tasks tended to be smaller than the N200 for words (data not shown).

\section{Auditory word-recognition task}

There was no detectable $\gamma$ power augmentation for auditory word-recognition tasks in the electrode recordings from the basal temporal area recorded on day 1 (Fig. $7 b$ ).

\section{Discussion}

This study recorded task-related broadband oscillatory activity from discrete areas in the basal temporal cortex during lexicaldecision and picture-naming tasks. The observed oscillatory activity was at a higher frequency than the usual definition of the $\gamma$ band, and we use the term high $\gamma$-band activity to collectively represent oscillatory activity $>60 \mathrm{~Hz}$. HGBA induced by the lexical-decision task was left dominant, whereas bilateral activity was seen during the naming tasks. A clear difference in the magnitude of HGBA in this brain region was observed for the two types of Japanese characters, being greater for kanji (morphogram) than for kana (syllabogram) stimuli. Significantly higher HGBA was observed in response to kanji pseudowords than to real kanji words. The picture-naming task induced a pattern of HGBA that was similar in the left hemisphere to and different in the right hemisphere from that induced by lexical-decision tasks. Time-frequency plots revealed task-related broadband GBA, which was especially prominent in the HGBA range. Although this study describes observations from a single case, our ERP findings reproduced those reported in a study evaluating many patients (Nobre et al., 1994) in terms of the location, timing, and lack of laterality for word stimuli. The excellent reproducibility of HGBA measurements and the high signal-to-noise ratio support the reliability of our data. The patient's epileptic focus was estimated to be in the left parahippocampal gyrus, which is rather close to the electrodes in which the task-related HGBA was observed. However, epileptic activity cannot account for the observed time-locked, task-specific oscillatory activity.

\section{Lexical-decision tasks}

The presence of oscillatory activity in the basal temporal area in response to kanji stimuli is consistent with previous findings, suggesting that kanji and kana represent two distinct graphemic codes that require different types of linguistic processing. In studies of brain-damaged Japanese patients, cases of pure kanji alexia (Kawamura et al., 1987) and pure kana alexia (Sakurai et al., 

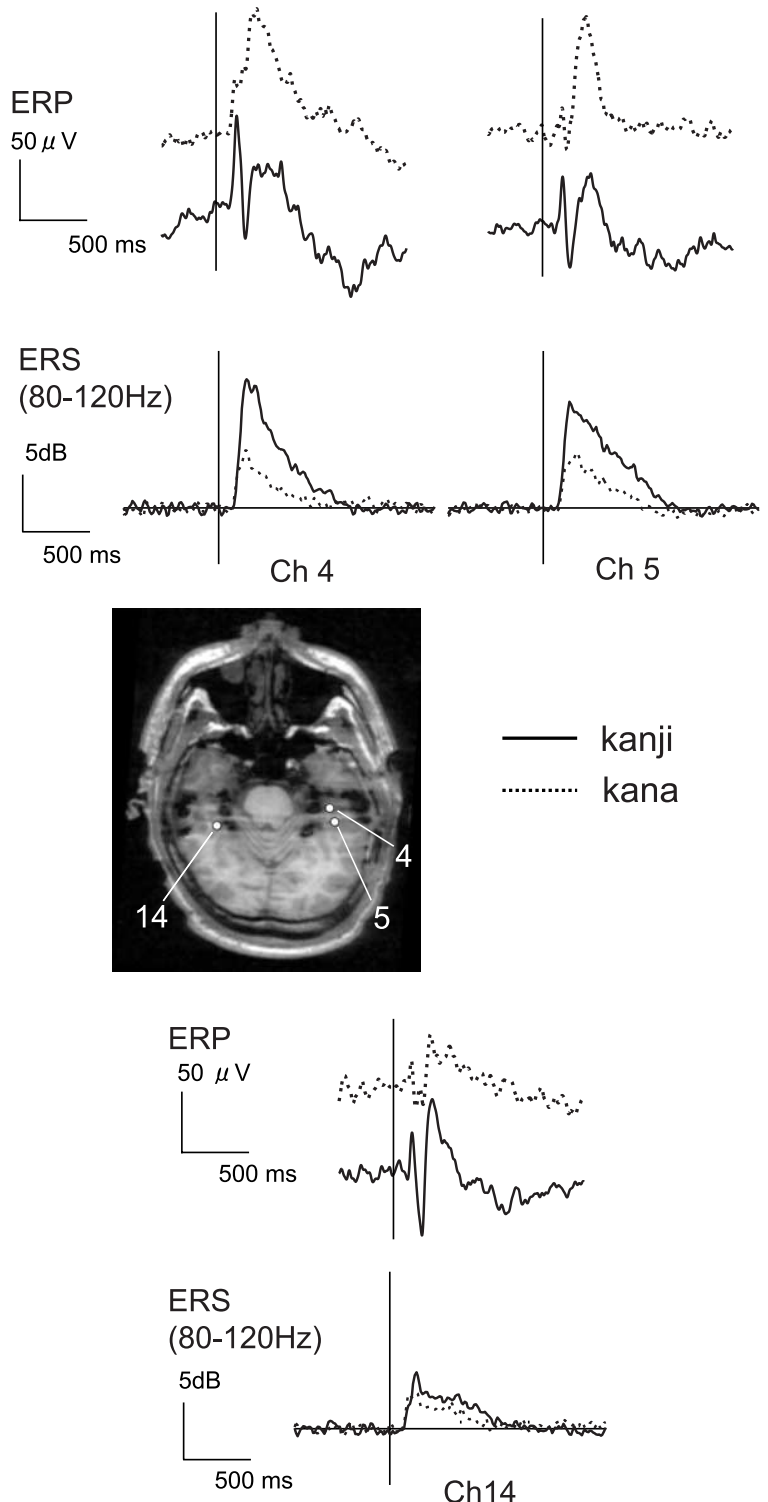

Figure 5. Comparison of ERP and ERS $(80-120 \mathrm{~Hz})$. A large negative peak of ERP at $\sim 200$ ms (N200) was observed bilaterally, most prominently for kanji. The N200 was less clear or absent with kana conditions. Interhemispheric dissociation of the power change was observed, which did not correlate with the amplitude of $\mathrm{N} 200$.

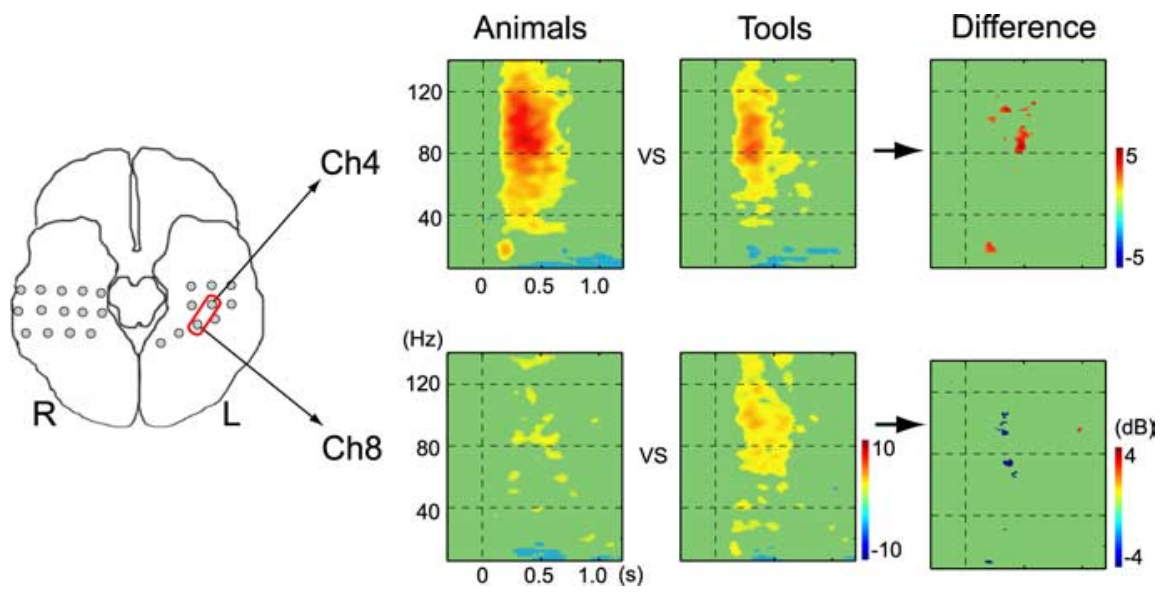

Figure 6. Comparison of ERSP plots between two categories in the naming task, for animals and tools. Greater HGBA was observed for animals in channel 4 and for tools in channel 8 . Categorical differences were observed in HGBA $(60-120 \mathrm{~Hz})$. R, Right; L, left.
2001) have been reported, indicating that the two systems are categorically different. One explanation for the ERSP difference through $\alpha$ to high- $\gamma$ band between kanji and kana (Fig. $3 a$ ) is that this reflects the difference in their visual properties; unlike the rather simple shapes of kana characters, kanji characters tend to include many strokes and consist of various radicals, or functional units, that may require not only finer visual structural analysis but also binding between the components. In addition to the visual differences, other factors could underlie the differential responses. In contrast to reading kana, in which there is an almost complete one-to-one correspondence of grapheme to syllable, reading kanji might require special processing mechanisms. Approximately 2000 kanji characters in regular use must be memorized by rote, and the kanji system lacks rules for spelling-tosound correspondences. Moreover, most kanji characters have several alternative phonological readings. The phonological reading of a kanji character is determined by the meaning of the character in a given context, which must be understood before the correct reading can be formulated (Sasanuma, 1980). In alphabetic systems, even those with irregular orthography, such as English, most words are regular in that their pronunciations adhere to standard spelling-sound correspondences (Plaut et al., 1996). However, a finding similar to ours was reported in a neuroimaging study of alphabetic writing systems with different degrees of spelling consistency. A difference in the levels of activation of the left fusiform area was observed when Italian and English readers performed a word-reading task (Paulesu et al., 2000). English readers showed greater activation in this brain area, and it was proposed that this reflected the need for a lexical/ semantic guide to decide the correct phonology in English, whereas Italian has a more regular orthography that does not require this step (Fiez, 2000). The divergence of broadband GBA between kanji and kana might reflect a similar process.

Although a detailed discussion of the $\alpha$-band is beyond the scope of this paper, it is noteworthy that there were significant increases in localized $\alpha$ - (and lower $\beta$-) band activity, specifically in response to kanji. This is consistent with a recent report that showed that $\alpha$-band oscillations do not necessarily represent brain "idling” (Jensen et al., 2002).

\section{Pseudowords and words}

Interestingly, kanji pseudowords induced greater HGBA than did real kanji words. Unlike the comparison of kanji and kana, this dissociation cannot be explained in terms of their visual property, because the words and pseudowords consisted of common kanji characters. Although the observed differences are consistent with recent findings in functional imaging studies comparing real alphabetic words and alphabetic pseudowords (Mechelli et al., 2003), this may seem to contradict a previous report that showed a stronger response to real words in the lower-frequency $\gamma$-band, at $\sim 30 \mathrm{~Hz}$ (Lutzenberger et al., 1994). These seemingly paradoxical activations might reflect an attempt to reach a semantic and contextual integration, which supports the implementation of phonetic values, rather than retrieval of meaning per se. Supporting this, a comparison of real kana words and kana pseudowords revealed no significant differences. Auditory word recogni- 


\section{Day 1}

\section{(a) Naming and Lexical decision}

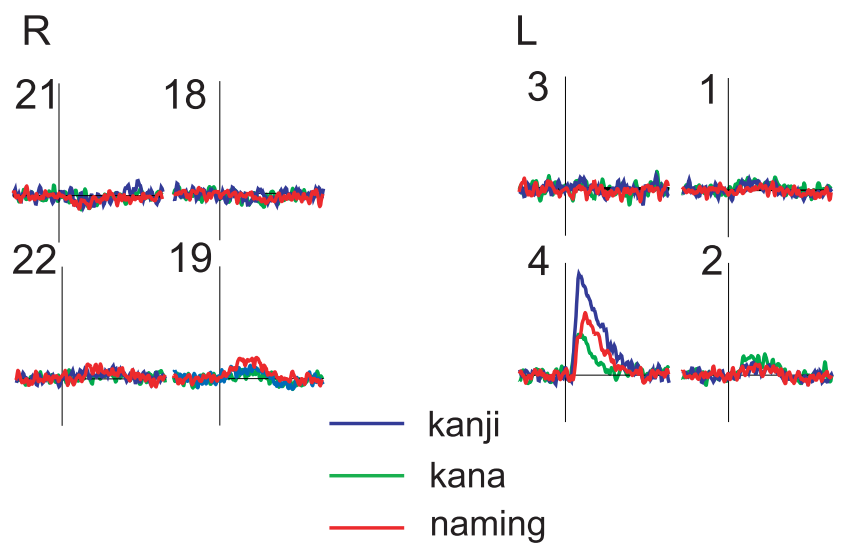

(b) Auditory word recognition

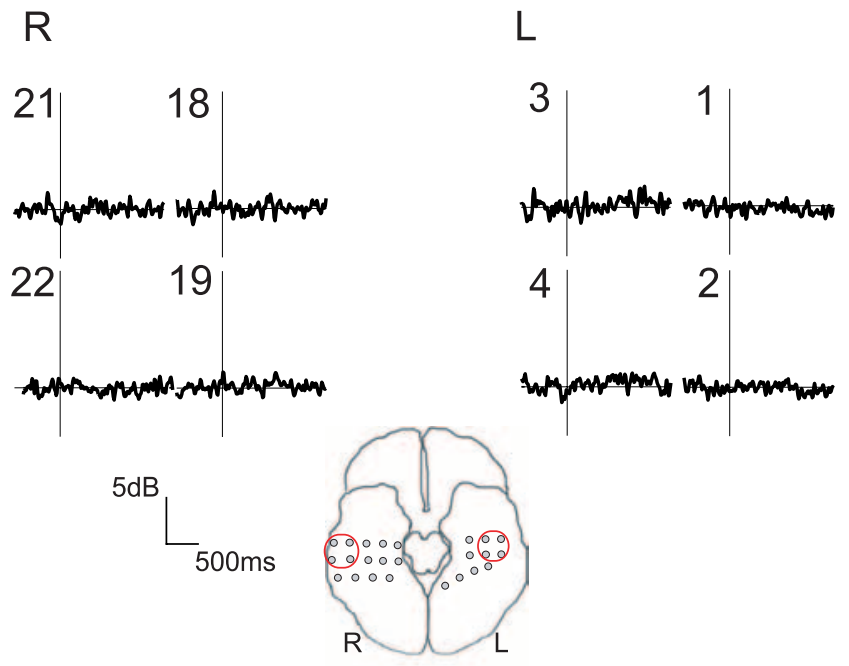

Figure 7. Overall view of the power change $(80-120 \mathrm{~Hz})$ recorded from electrodes on day 1. The channel numbers are the same as in Figure 1. $\boldsymbol{a}$, Naming and lexical-decision tasks showed power changes similar to those recorded on day 2. $\boldsymbol{b}$, Auditory word-recognition tasks did not show notable power changes. R, Right; L, left.

tion also caused no activation in the basal temporal area. These results suggest that this area is not specialized for semantic processing. This agrees with Damasio et al. (1996), who proposed that the basal temporal area plays a mediational role in lexical retrieval.

\section{Picture-naming task}

Despite the large differences in the visual properties of words and of pictures of objects, HGBA was commonly observed at the same two electrodes in the left hemisphere in both reading and naming tasks. This is consistent with the finding that patients with kanji alexia also frequently have naming disorders (Sakurai et al., 2000). In patients with large temporal lesions, comprehension of kanji and the ability to read them sometimes dissociate (Yamawaki et al., 2005). A similar finding reported that electrical stimulation of the left basal temporal area selectively disrupted kanji reading and object naming but not kana reading (Usui et al., 2003). Importantly, the subject could comprehend the meaning of the presented objects and kanji words. These findings suggest that kanji reading and object naming share common neural substrates linking concept to phonemic representation, which plays a mediational role in lexical retrieval (Damasio et al., 1996). The double dissociation of HGBA accompanying the naming of animals and tools that we observed agrees with previous reports of category-specific functional localization in the basal temporal area (Damasio et al., 1996; Chao et al., 1999).

\section{The basal temporal area and high-frequency oscillatory activity}

It has been proposed that the fusiform area is homologous to the inferotemporal (IT) area in the monkey, because face-selective responses can be recorded from both areas (Tootell et al., 2003). The IT area shows visual-response properties that are distinct from those in evolutionarily older cortical areas (Fujita, 2002) and has been proposed to serve as an "expertise center." Cohen et al. (2000) proposed that there is a unimodal visual word-form area (VWFA) in the left fusiform area that has perceptual expertise in sublexical processing of visual word forms (McCandliss et al., 2003). The present study revealed localized electrophysiological activity in response to visual words, with virtually no observed response to auditory words. The notion of VWFA, however, cannot fully explain our data. First, HGBA was observed in both picture-naming tasks and lexical-decision tasks. Second, although the kana words used did not violate the lettercombination rules, they induced consistently less HGBA than did kanji words, which cannot be accounted for by sublexical wordform processing in this brain area. Third, the divergence seen between kanji words and kanji pseudowords should not occur if this brain area specializes in sublexical analysis, because the words and pseudowords used consisted of common kanji characters. Reading is a distributed process, and it is unlikely that visual word-form representations are subtended solely by a single region of the cortex (Price and Devlin, 2003). The IT area interconnects with many cortical structures and may therefore integrate multiple aspects of vision and relay information to areas required for kanji word processing. This would involve many processes, such as visual character segmentation within words, association of visual representation with semantic processes, and selection of phonetic values, depending on contextual information. Therefore, this area is better characterized as a "convergence zone" (Damasio, 1989), in which feedforward and feedback neural loops make contact. It has been proposed that high-frequency oscillatory activity mediates the signal for reciprocal connection, binding together the areas involved in object representation (Singer, 1993). High- $\gamma$ activity seems optimal for this role because of its high temporal resolution. The task-specific localized HGBA observed here is consistent with this proposal.

\section{References}

Allison T, Puce A, Spencer DD, McCarthy G (1999) Electrophysiological studies of human face perception. I. Potentials generated in occipitotemporal cortex by face and non-face stimuli. Cereb Cortex 9:415-430.

Blair RC, Karniski W (1993) An alternative method for significance testing of waveform difference potentials. Psychophysiology 30:518-524.

Bonferroni CE (1950) Sulle medie multiple di potenze. Boll Unione Mat Ital 5:267-270.

Breier JI, Simos PG, Zouridakis G, Wheless JW, Willmore LJ, Constantinou JEC, Maggio WW, Papanicolaou AC (1999) Language dominance determined by magnetic source imaging: a comparison with the Wada procedure. Neurology 53:938-945.

Chao LL, Haxby JV, Martin A (1999) Attribute-based neural substrates in temporal cortex for perceiving and knowing about objects. Nat Neurosci 2:913-919.

Cohen L, Dehaene S, Naccache L, Lehericy S, Dehaene-Lambertz G, Henaff 
M-A, Michel F (2000) The visual word form area: spatial and temporal characterization of an initial stage of reading in normal subjects and posterior split-brain patients. Brain 123:291-307.

Crone N, Miglioretti D, Gordon B, Lesser R (1998) Functional mapping of human sensorimotor cortex with electrocorticographic spectral analysis. II. Event-related synchronization in the gamma band. Brain 121:2301-2315.

Crone NE, Boatman D, Gordon B, Hao L (2001) Induced electrocorticographic gamma activity during auditory perception. Brazier Awardwinning article, 2001. Clin Neurophysiol 112:565-582.

Damasio AR (1989) The brain binds entities and events by multiregional activation from convergence zones. Neural Comput 1:123-132.

Damasio H, Grabowski TJ, Tranel D, Hichwa RD, Damasio AR (1996) A neural basis for lexical retrieval. Nature 380:499-505.

Delorme A, Makeig S (2004) EEGLAB: an open source toolbox for analysis of single-trial EEG dynamics including independent component analysis. J Neurosci Methods 134:9-21.

Eckhorn R, Frien A, Bauer R, Woelbern T, Kehr H (1993) High frequency $(60-90 \mathrm{~Hz})$ oscillations in primary visual cortex of awake monkey. NeuroReport 4:243-246.

Fell J, Klaver P, Lehnertz K, Grunwald T, Schaller C, Elger CE, Fernandez G (2001) Human memory formation is accompanied by rhinalhippocampal coupling and decoupling. Nat Neurosci 4:1259-1264.

Fiez JA (2000) Sound and meaning: how native language affects reading strategies. Nat Neurosci 3:3-5.

Fries P, Roelfsema PR, Engel AK, König P, Singer W (1997) Synchronization of oscillatory responses in visual cortex correlates with perception in interocular rivalry. Proc Natl Acad Sci USA 94:12699-12704.

Fries P, Reynolds JH, Rorie AE, Desimone R (2001) Modulation of oscillatory neuronal synchronization by selective visual attention. Science 291:1560-1563.

Fujita I (2002) The inferior temporal cortex: architecture, computation, and representation. J Neurocytol 31:359-371.

Gray CM, König P, Engel AK, Singer W (1989) Oscillatory responses in cat visual cortex exhibit inter-columnar synchronization which reflects global stimulus properties. Nature 338:334-337.

Gray CM, Engel AK, König P, Singer W (1992) Synchronization of oscillatory neuronal responses in cat striate cortex: temporal properties. Vis Neurosci 8:337-347.

Jensen O, Gelfand J, Kounios J, Lisman JE (2002) Oscillations in the alpha band $(9-12 \mathrm{~Hz})$ increase with memory load during retention in a shortterm memory task. Cereb Cortex 12:877-882.

Kawamura M, Hirayama K, Hasegawa K, Takahashi N, Yamaura A (1987) Alexia with agraphia of kanji (Japanese morphograms). J Neurol Neurosurg Psychiatry 50:1125-1129.

Lutzenberger W, Pulvermüller F, Birbaumer N (1994) Words and pseudowords elicit distinct patterns of 30-Hz EEG responses in humans. Neurosci Lett 176:115-118.

Makeig S (1993) Auditory event-related dynamics of the EEG spectrum and effects of exposure to tones. Electroencephalogr Clin Neurophysiol 86:283-293.

McCandliss BD, Cohen L, Dehaene S (2003) The visual word form area: expertise for reading in the fusiform gyrus. Trends Cogn Sci 7:293-299.
Mechelli A, Gorno-Tempini ML, Price CJ (2003) Neuroimaging studies of word and pseudoword reading: consistencies, inconsistencies, and limitations. J Cogn Neurosci 15:260-271.

Nichols TE, Holmes AP (2002) Nonparametric permutation tests for functional neuroimaging: a primer with examples. Hum Brain Mapp 15:1-25.

Nobre AC, Allison T, McCarthy G (1994) Word recognition in the human inferior temporal lobe. Nature 372:260-263.

Paulesu E, McCrory E, Fazio F, Menoncello L, Brunswick N, Cappa SF, Cotelli M, Cossu G, Corte F, Lorusso M, Pesenti S, Gallagher A, Perani D, Price C, Frith CD, Frith U (2000) A cultural effect on brain function. Nat Neurosci 3:91-96.

Pfurtscheller G, Aranibar A (1979) Evaluation of event-related desynchronization (ERD) preceding and following voluntary self-paced movement. Electroencephalogr Clin Neurophysiol 46:138-146.

Pfurtscheller G, Cooper R (1975) Frequency dependence of the transmission of the EEG from cortex to scalp. Electroencephalogr Clin Neurophysiol 38:93-96.

Plaut DC, McClelland JL, Seidenberg MS, Patterson K (1996) Understanding normal and impaired word reading: computational principles in quasi-regular domains. Psychol Rev 103:56-115.

Price CJ, Devlin JT (2003) The myth of the visual word form area. NeuroImage 19:473-481.

Ramberg JS, Dudewicz EJ, Tadikamalla PR, Mykytka EF (1979) A probability distribution and its uses in fitting data. Technometrics 21:201-214.

Sakurai Y, Sakai K, Sakuta M, Iwata M (1994) Naming difficulties in alexia with agraphia for kanji after a left posterior inferior temporal lesion. J Neurol Neurosurg Psychiatry 57:609-613.

Sakurai Y, Takeuchi S, Takada T, Horiuchi E, Nakase H, Sakuta M (2000) Alexia caused by a fusiform or posterior inferior temporal lesion. J Neurol Sci 178:42-51.

Sakurai Y, Ichikawa Y, Mannen T (2001) Pure alexia from a posterior occipital lesion. Neurology 56:778-781.

Sasanuma S (1980) Acquired dyslexia in Japanese: clinical features and underlying mechanisms. In: Deep dyslexia (Coltheart M, Patterson K, Marshall JC, eds), pp 48-90. London: Routledge and Kegan Paul.

Singer W (1993) Synchronization of cortical activity and its putative role in information processing and learning. Annu Rev Physiol 55:349-374.

Tokunaga H, Nishikawa T, Ikejiri Y, Nakagawa Y, Yasuno F, Hashikawa K, Nishimura T, Sugita Y, Takeda M (1999) Different neural substrates for kanji and kana writing: a PET study. NeuroReport 10:3315-3319.

Tootell RBH, Tsao D, Vanduffel W (2003) Neuroimaging weighs in: humans meet macaques in "primate" visual cortex. J Neurosci 23:3981-3989.

Usui K, Ikeda A, Takayama M, Matsuhashi M, Yamamoto J-I, Satoh T, Begum T, Mikuni N, Takahashi JB, Miyamoto S, Hashimoto N, Shibasaki H (2003) Conversion of semantic information into phonological representation: a function in left posterior basal temporal area. Brain 126:632-641.

Yamawaki R, Suzuki K, Tanji K, Fujii T, Endo K, Meguro K, Yamadori A (2005) Anomic alexia of kanji in a patient with anomic aphaisa. Cortex, in press. 